\title{
On nonmeasurable selectors of countable group actions
}

by

\author{
Piotr Zakrzewski (Warszawa)
}

\begin{abstract}
Given a set $X$, a countable group $H$ acting on it and a $\sigma$-finite $H$-invariant measure $m$ on $X$, we study conditions which imply that each selector of $H$-orbits is nonmeasurable with respect to any $H$-invariant extension of $m$.
\end{abstract}

1. Introduction. Let $H$ be a group of permutations of a set $X$. An $H$-orbit is the set $H x=\{h(x): h \in H\}$. A complete section for $H$ is a subset of $X$ that meets every $H$-orbit. A selector of $H$-orbits (sometimes also called a Vitali set of $H$, see [13]) is a subset of $X$ that meets every $H$-orbit in exactly one point. A $\sigma$-algebra $\mathcal{A}$ of subsets of $X$ is $H$-invariant if $h[A] \in \mathcal{A}$ for every $A \in \mathcal{A}$ and $h \in H$; then we also say that $H$ is a group of measurable transformations of $X$. By a measure on $X$ we mean a $\sigma$-additive, nonzero measure $m: \mathcal{A} \rightarrow[0, \infty]$ defined on a $\sigma$-algebra of subsets of $X$. We say that $m$ is $H$-invariant if $\mathcal{A}$ is $H$-invariant and $m(h[A])=m(A)$ for every $A \in \mathcal{A}$ and $h \in H$.

Recall that every Vitali set of the group $\mathbb{Q}$ of rational translations of $\mathbb{R}$, the set of reals, is nonmeasurable with respect to any $\mathbb{Q}$-invariant extension of the Lebesgue measure on $\mathbb{R}$. This situation is to some extent typical. Indeed, Solecki [14] proved that if a $\sigma$-finite measure $m$ is invariant with respect to an uncountable group $G$ of permutations of $X$ and, moreover, the action of $G$ is $m$-free (i.e., $m^{*}(\{x \in X: g(x)=x\})=0$ for any $g \in G \backslash\left\{\operatorname{id}_{X}\right\}$, where $m^{*}$ stands for the outer measure of $m$ ), then there exists a countable subgroup $H$ of $G$ such that each Vitali set of $H$ is nonmeasurable with respect to any $H$-invariant extension of $m$.

Throughout the paper we always assume that $m: \mathcal{A} \rightarrow[0, \infty]$ is a $\sigma$-finite measure on $X$, invariant with respect to a countable group $H$ of measur-

2000 Mathematics Subject Classification: Primary 28A05, 28C10; Secondary 28D05.

Key words and phrases: invariant measure, countable group of transformations, complete section of orbits, selector of orbits, countably equidecomposable sets, nonmeasurable sets, Borel measure. 
able transformations of $X$. Following [5], the quadruple $\langle X, \mathcal{A}, H, m\rangle$ will sometimes be referred to as a dynamical system.

In principle, there are two methods of proving that selectors of $H$-orbits are nonmeasurable:

1. The Vitali method. The original Vitali [16] method was further elaborated by Solecki [13], [14] and, in particular, used by him in the proof of the theorem quoted above. Let us say that the measure $m$ (more precisely: the dynamical system $\langle X, \mathcal{A}, H, m\rangle)$ satisfies the Vitali condition $(\mathbf{V})$ if there exists a complete section $A \in \mathcal{A}$ for $H$ such that

$$
0<m(A)<\infty \text { and } m^{*}(\{x:|H x \cap A|<\omega\})=0 .
$$

Note that if $H=\left\{h_{n}: n \in \mathbb{N}\right\}$ with $h_{n} \neq h_{m}$ for $n \neq m$, then for every $x \in X$, if $H x \cap A$ is infinite, then $x \in \bigcap_{m=0}^{\infty} \bigcup_{n>m} h_{n}^{-1}[A]$, and the converse is also true provided that $h_{n}(x) \neq h_{m}(x)$ for $n \neq m$. It follows that if the action of $H$ is $m$-free then in the terminology of [13] condition ( $\mathbf{V})$ means that $X$ is infinitely covered by $H$, up to a subset of outer measure zero. The following fact was proved in [13, Lemma 3.1] under the assumption that the action of $H$ is $m$-free. A proof not using this assumption will be given at the beginning of Section 2.

Proposition 1.1. Let $H$ be a countable group of permutations of a set $X$ and let $m: \mathcal{A} \rightarrow[0, \infty]$ be a $\sigma$-finite $H$-invariant measure on $X$. If $m$ satisfies the Vitali condition, then every selector of $H$-orbits is nonmeasurable with respect to any $H$-invariant extension of $m$.

More applications of condition ( $\mathbf{V})$ may be found in [13] and [14].

2. The Minkowski method. This is a method invented by Minkowski [11] for another purpose and elaborated by Kharazishvili and Kirtadze in their recent paper [8] in order to demonstrate its role in existence proofs of nonmeasurable sets for invariant measures. Let us say that the measure $m$ (more precisely: the dynamical system $\langle X, \mathcal{A}, H, m\rangle)$ satisfies the Minkowski condition $(\mathbf{M})$ if for every $\varepsilon>0$ there exists a complete section $A \in \mathcal{A}$ for $H$ such that $m(A)<\varepsilon$. In the terminology of [8], condition (M) means that the measure $m$ is weakly metrically transitive, and in the terminology of [5], this is equivalent to the statement that the dynamical system $\langle X, \mathcal{A}, H, m\rangle$ is continuous. Examples of measures satisfying (M) include the Haar measure on an uncountable locally compact Polish group $X$, where $H$ is a countable nondiscrete subgroup of $X$ acting on it by (say) left shifts (see [8, Theorem 3]), and the $n$-dimensional Lebesgue measure on $X=\mathbb{R}^{n}$, where $H$ is a countable, nondiscrete group of isometries of $\mathbb{R}^{n}$ (see $[8$, Theorem 4]).

The following fact was proved in [8, Theorem 2], again under the additional assumption that the action of $H$ is $m$-free. A proof avoiding this assumption will also be given at the beginning of Section 2 . 
Proposition 1.2. Let $H$ be a countable group of permutations of a set $X$ and let $m: \mathcal{A} \rightarrow[0, \infty]$ be a $\sigma$-finite $H$-invariant measure on $X$. If $m$ satisfies the Minkowski condition, then every selector of $H$-orbits is nonmeasurable with respect to any $H$-invariant extension of $m$.

The aim of this paper is to compare the strength of conditions (V) and (M) and to evaluate their role in proving that all selectors of $H$-orbits are nonmeasurable with respect to any $H$-invariant extension of $m$. We observe that (M) always implies (V) (Theorem 2.2) (evidently, in general, not vice versa). On the other hand, if $H$ is a countable group of Borel automorphisms of a Polish space $X$ and $m$ is an $H$-invariant $\sigma$-finite Borel measure on $X$ (i.e., $m$ is defined on the $\sigma$-algebra $\mathbf{B}(X)$ of Borel subsets of $X$ ), then the two conditions are equivalent (Theorem 3.1) and their local instances are equivalent to the statement that every selector of $H$-orbits is nonmeasurable with respect to every $H$-invariant extension of $m$ (Theorem 3.2). We also give an example showing that the latter is not true in general (Example 4.1) but for reasons that have not much to do with the group $H$ under consideration. Indeed, under a certain condition imposed on the measure space $\langle X, \mathcal{A}, m\rangle$ we prove that a local instance of condition $(\mathbf{V})$ is in fact necessary even for the existence of a selector of $H$-orbits, nonmeasurable with respect to every $H$-invariant extension of $m$ (Theorem 4.4); the latter is also true if the action of $H$ is $m$-free (Theorem 4.6). The results just quoted are consequences of the main technical result of the paper (Theorem 4.3) giving sufficient conditions for the existence of an $H$-invariant extension of $m$ for which a given selector of $H$-orbits is measurable.

Our main technical notion is countable equidecomposability. All facts that we use concerning this notion may be retrieved from a well-forgotten paper of Kawada [6] (see also [2], [5] and [19]).

We use standard set-theoretical notation. In particular, the cardinality of a set $A$ is denoted by $|A|$. The first infinite cardinal is the first infinite ordinal $\omega$ and we identify it with the set of natural numbers; thus $|A|<$ $\omega$ (respectively: $|A|=\omega$ ) means that $A$ is finite (respectively: countable infinite) and $n<\omega$ means that $n$ is a natural number.

2. (M) versus (V) for general actions. Recall that $m: \mathcal{A} \rightarrow[0, \infty]$ is a $\sigma$-finite measure on a set $X$ and $m$ is invariant with respect to a countable group $H$ of measurable transformations of $X$.

We say that sets $A, B \in \mathcal{A}$ are countably $H$-equidecomposable in $\mathcal{A}$, in symbols $A \sim_{\infty} B$ in $\mathcal{A}$, if there is a partition of $A$ into countably many sets $A_{n} \in \mathcal{A}, n<\omega$, and elements $h_{n} \in H$ such that the sets $h_{n}\left[A_{n}\right]$ form a partition of $B$. If $\mathcal{A}$ and $H$ are clear from the context we simply say that $A$ and $B$ are countably equidecomposable and write $A \sim_{\infty} B$. 
We say that sets $A, B \in \mathcal{A}$ are almost countably equidecomposable in $\mathcal{A}$, and write $A \stackrel{m}{\sim} \infty B$ in $\mathcal{A}$, if there are $E_{1}, E_{2} \in \mathcal{A}$ such that $m\left(E_{1}\right)=$ $m\left(E_{2}\right)=0$ and $A \backslash E_{1} \sim_{\infty} B \backslash E_{2}$ in $\mathcal{A}$. Clearly, if $A, B \in \mathcal{A}$ and $A \stackrel{m}{\sim} B$, then $m(A)=m(B)$.

A set $C \subseteq X$ is $H$-invariant if $h[C]=C$ for every $h \in H$; the smallest $H$-invariant set containing $A \subseteq X$ is $A^{*}=\bigcup_{h \in H} h[A]$.

We call sets $A, B \in \mathcal{A}$ almost equal and write $A \stackrel{m}{=} B$ if $m(A \triangle B)=0$. An almost complete section for $H$ is a set $A \subseteq X$ such that $A^{*} \stackrel{m}{=} X$; likewise, $S$ is an almost selector of $H$-orbits if $S^{*} \stackrel{m}{=} X$ and $S$ meets every $H$-orbit in at most one point. Note that $A \stackrel{m}{=} B$ (respectively $A \stackrel{m}{\sim} B$ in $\mathcal{A}$ ) if and only if there is an $H$-invariant set $C \in \mathcal{A}$ such that $C \stackrel{m}{=} X$ and $A \cap C=B \cap C$ (respectively $A \cap C \sim_{\infty} B \cap C$ in $\mathcal{A}$ ).

Clearly, condition $(\mathbf{V})$ states the existence of an almost complete section $A \in \mathcal{A}$ for $H, 0<m(A)<\infty$, having infinite intersection with every $H$-orbit it meets.

The following folklore-like fact is a special case of the comparability theorem (see e.g. [6] or [5]).

Lemma 2.1 (The comparability lemma). If $A, B \in \mathcal{A}$ are almost complete sections for $H$, then there is an $H$-invariant set $Z \in \mathcal{A}$ such that $A \cap Z$ is almost countably equidecomposable in $\mathcal{A}$ with a subset of $B$ and $B \backslash Z$ is almost countably equidecomposable in $\mathcal{A}$ with a subset of $A$. In particular, if $S \in \mathcal{A}$ is an almost selector of $H$-orbits and $A \in \mathcal{A}$ is an almost complete section for $H$-orbits, then $S$ is almost countably equidecomposable in $\mathcal{A}$ with a subset of $A$.

Proofs of Propositions 1.1 and 1.2 are now almost immediate.

Proof of Proposition 1.1. Let $S$ be an arbitrary selector of $H$-orbits and let $A \in \mathcal{A}$ be an almost complete section for $H$ witnessing condition (V). Suppose that $m^{\prime}: \mathcal{A}^{\prime} \rightarrow[0, \infty]$ is an $H$-invariant extension of $m$ and $S \in \mathcal{A}^{\prime}$. By the comparability lemma, $S$ is almost countably equidecomposable in $\mathcal{A}^{\prime}$ with a subset of $A$; moreover, an easy induction shows that there is an infinite sequence $\left\langle S_{n}: n<\omega\right\rangle$ of pairwise disjoint almost selectors of $H$ orbits such that $S_{n} \subseteq A$ and $S_{n} \stackrel{m}{\sim} S$ for each $n<\omega$. This easily implies that $m^{\prime}(S)=0$, a contradiction.

Proof of Proposition 1.2. Let $m^{\prime}: \mathcal{A}^{\prime} \rightarrow[0, \infty]$ be an $H$-invariant extension of $m$. Let $S$ be a selector of $H$-orbits and suppose that $S \in \mathcal{A}^{\prime}$. Take an arbitrary $\varepsilon>0$ and let $A \in \mathcal{A}$ be a complete section for $H$ with $m(A)<\varepsilon$. By the comparability lemma, $S$ is almost countably equidecomposable in $\mathcal{A}^{\prime}$ with a subset of $A$. Hence $m^{\prime}(S)<\varepsilon$. But due to the arbitrary choice of $\varepsilon>0$ this implies that $m^{\prime}(S)=0$, a contradiction. 
Now we are ready to state the main result of this section.

THEOREM 2.2. Let $H$ be a countable group of permutations of a set $X$ and let $m: \mathcal{A} \rightarrow[0, \infty]$ be a $\sigma$-finite $H$-invariant measure on $X$. If $m$ satisfies the Minkowski condition then it satisfies the Vitali condition as well.

Proof. Assume that $m$ satisfies condition (M). Then it follows from a lemma of Kawada (see [6, Lemma 19] or [5, Corollary V.3]) that every almost complete section for $H$ can be partitioned into two disjoint almost complete sections for $H$.

Using this, inductively construct a sequence $\left\langle A_{n}: n<\omega\right\rangle$ of pairwise disjoint almost complete sections for $H$ keeping the following conditions satisfied for every $n<\omega$ :

- $A_{n} \in \mathcal{A}$ and $m\left(A_{n}\right)<1 / 2^{n+1}$,

- $X \backslash \bigcup_{k<n} A_{k}$ is a complete section for $H$.

Finally, $A=\bigcup_{n<\omega} A_{n}$ is an almost complete section for $H$ witnessing condition $(\mathbf{V})$.

It is obvious that the implication from the theorem above cannot in general be reversed. A trivial example is provided by an arbitrary infinite set $X$ together with a countable group $H$ of permutations of $X$ with infinite $H$-orbits, the $\sigma$-algebra $\mathcal{A}=\{\emptyset, X\}$ and the canonical two-valued probability measure $m$ defined on $\mathcal{A}$.

3. (M) versus (V) for Borel actions. Natural examples of measures satisfying condition (M) happen to be of the form: a countable group $H$ of Borel automorphisms of an uncountable Polish (i.e., separable, completely metrizable) space $X$ and an $H$-invariant $\sigma$-finite Borel measure $m$ on $X$. Recall that they include the Haar measure on an uncountable locally compact Polish group $X$ (where $H$ is a countable nondiscrete subgroup of $X$ ) and the $n$-dimensional Lebesgue measure on $\mathbb{R}^{n}$ (where $H$ is a countable, nondiscrete group of isometries of $\mathbb{R}^{n}$ ). Moreover, Kharazishvili and Kirtadze proved (see [8, Theorems 3 and 4]) that in these cases condition (M) is equivalent to the statement that every selector of $H$-orbits is nonmeasurable with respect to any $H$-invariant extension of $m$. The results of this section generalize the latter to the case of arbitrary Borel measures on Polish spaces and also show that in this situation conditions $(\mathbf{M})$ and $(\mathbf{V})$ are equivalent.

Recall that the partition into $H$-orbits defines an equivalence relation $E_{H}$ on $X$ called the orbit equivalence relation. If $B \subseteq X$, then $E_{H} \mid B$ denotes the restriction of $E_{H}$ to $B$, i.e., the equivalence relation on $B$ whose equivalence classes are exactly the nonempty intersections of $H$-orbits with $B$. 
If $E$ is an equivalence relation on a set $B \subseteq X$, we say that a subset of $B$ is:

- a complete section for $E$ if it meets every $E$-equivalence class,

- a selector for $E$ if it meets every $E$-equivalence class in exactly one point,

- a partial selector for $E$ if it meets every $E$-equivalence class in at most one point.

Note that if $B=X$ and $E=E_{H}$, then the notions of a complete section and a selector for $E_{H}$ coincide with those for $H$.

THEOREM 3.1. Let $H$ be a countable group of Borel automorphisms of an uncountable Polish space $X$ and let $m$ be an $H$-invariant $\sigma$-finite Borel measure on $X$. Then the following conditions are equivalent:

(1) $m$ satisfies the Minkowski condition,

(2) $m$ satisfies the Vitali condition,

(3) $m$ vanishes on every partial Borel selector for $E_{H}$.

Proof. By Theorem 2.2, (1) implies (2). To see that (2) implies (3), argue as in the proof of Proposition 1.1.

So assume now that $m$ vanishes on every partial Borel selector for $E_{H}$. Since $m$ is $\sigma$-finite, $X$ is a disjoint union of countably many sets of the form $B^{*}$ where $B$ is a Borel set with $0<m(B)<\infty$. Then the union of small enough complete sections for equivalence relations $E_{H} \mid B$ will be a complete section for $H$ we are looking for. Thus, with no loss of generality, we simply assume that $X=B^{*}$ for a fixed $B \in \mathbf{B}(X)$ with $0<m(B)<\infty$. Let $E=E_{H} \mid B$ and $\varepsilon>0$.

Let $C=\{x \in B:|H x \cap B|<\omega\}$. Since $C \in \mathbf{B}(X)$ and $E \mid C$ is a Borel equivalence relation with finite equivalence classes, there is a Borel selector $S \subseteq C$ for $E \mid C$ (see [7, Theorem 12.16]). Then $S$ is a partial selector for $E_{H}$, so $m(S)=0$, and since $C \subseteq \bigcup_{h \in H} h[S]$, we have $m(C)=0$ as well.

Let $D=B \backslash C$. Then $E \mid D$ is a Borel equivalence relation on $D$ with countable infinite equivalence classes, and a basic fact about such relations says (see [3, Lemma 4.5.3]) that there is a sequence $D=A_{0} \supseteq A_{1} \supseteq \ldots$ of Borel complete sections for $E \mid D$ such that $\bigcap_{n<\omega} A_{n}=\emptyset$. Since $m(D)=$ $m(B)<\infty$ it follows that there is $n_{0}$ for which $m\left(A_{n_{0}}\right)<\varepsilon$. Then $A=$ $A_{n_{0}} \cup S$ is a complete section for $H$ with $m(A)<\varepsilon$. This completes the proof of the implication $(3) \Rightarrow(1)$.

As a corollary we show that local instances of conditions $(\mathbf{V})$ and $(\mathbf{M})$ are in fact also necessary for the nonmeasurability of every selector of $H$-orbits with respect to all $H$-invariant extensions of the $H$-invariant Borel measure $m$ under consideration. 
THEOREM 3.2. Let $H$ be a countable group of Borel automorphisms of an uncountable Polish space $X$ and let $m$ be an $H$-invariant $\sigma$-finite Borel measure on $X$. Then the following conditions are equivalent:

(1) there is $B \in \mathbf{B}(X)$ such that $0<m(B)<\infty$ and for every $\varepsilon>$ 0 there exists a complete section $A \in \mathbf{B}(X)$ for $E_{H} \mid B$ such that $m(A)<\varepsilon$,

(2) there is $B \in \mathbf{B}(X)$ such that $0<m(B)<\infty$ and $|H x \cap B|=\omega$ for every $x \in B$,

(3) there is $B \in \mathbf{B}(X)$ such that $0<m(B)<\infty$ and $m$ vanishes on every partial Borel selector for $E_{H} \mid B$,

(4) every selector of $H$-orbits is nonmeasurable with respect to any $H$ invariant extension of $m$,

(5) every selector of $H$-orbits is nonmeasurable with respect to the measure completion $\bar{m}$ of $m$,

(6) there is no Borel almost selector of $\mathrm{H}$-orbits.

Proof. The equivalence of the first three conditions is just a relativization of Theorem 3.1. The fact that any of the first two of them implies (4) follows from Propositions 1.2 and 1.1. The implications $(4) \Rightarrow(5) \Rightarrow(6)$ are obvious. Finally, if every Borel subset $B$ of positive finite measure contains a partial Borel selector for $E_{H} \mid B$ of positive measure, then it is easy to construct a Borel almost selector of $H$-orbits. This shows that $\neg(3)$ implies $\neg(6)$, completing the proof.

REMARK 3.3. Let $H$ be a countable group of Borel automorphisms of an uncountable Polish space $X$. There exists an $H$-invariant $\sigma$-finite Borel measure $m$ on $X$ with the properties from Theorem 3.2 if and only if there exists a Borel subset $B$ of $X$ satisfying the following conditions:

- $|H x \cap B|=\omega$ for every $x \in B$,

- $B$ is incompressible, i.e., there is no Borel set $A \subseteq B$ with $B \sim_{\infty} A$ in $\mathbf{B}(X)$ and such that $B \backslash A$ is a complete section for $E_{H} \mid B$.

Proof. First assume that $m$ is an $H$-invariant $\sigma$-finite Borel measure on $X$ satisfying condition (2) of Theorem 3.2. Take $B \in \mathbf{B}(X)$ with $0<m(B)<$ $\infty$ and $|H x \cap B|=\omega$ for every $x \in B$. Clearly, $B$ is incompressible. This shows the necessity of the conditions above. To prove their sufficiency, fix an incompressible set $B \in \mathbf{B}(X)$ with $|H x \cap B|=\omega$ for every $x \in B$. Since $B$ is incompressible, by Nadkarni's theorem (see [12] and [3, Theorem 4.3.1] for a proof in the general case), there exists a probability Borel measure $\mu$ on $B$ which is $H$-invariant in the following sense: whenever $A_{1}, A_{2} \in \mathbf{B}(B)$ and $A_{1} \sim_{\infty} A_{2}$ in $\mathbf{B}(X)$, then $\mu\left(A_{1}\right)=\mu\left(A_{2}\right)$. Now it is easy to extend $\mu$ to a $\sigma$-finite $H$-invariant Borel measure $m$ on $X$ (see e.g. [18, Proposition $2.2])$; in particular, $0<m(B)=\mu(B)<\infty$. 
4. The necessity of a local instance of (V) for the existence of nonmeasurable selectors. It is obvious that in general, by contrast to the Borel case discussed in the previous section, no local instance of condition (M) follows from the statement that every selector of $H$-orbits is nonmeasurable with respect to any $H$-invariant extension of $m$ (see the last paragraph of Section 2). We start this section with an example in which the latter is still true but all $H$-orbits are finite (in particular, no local instance of condition ( $\mathbf{V})$ is satisfied).

Recall that $Y \subseteq \mathbb{R}$ is a universally measure zero set if it carries no finite nonzero Borel measure vanishing on singletons (see e.g. [10]).

EXAMPLE 4.1. There exist a set $X$ and a probability measure $m: \mathcal{A} \rightarrow[0,1]$ invariant with respect to a countable group $H$ of permutations of $X$ and having the following properties:

- every selector of $H$-orbits is nonmeasurable with respect to any $H$ invariant extension of $m$,

- $|H x|=2$ for every $x \in X$.

Proof. Let $Y \subseteq \mathbb{R}$ be an uncountable universally measure zero set. Let $\mathcal{U}=\left\{U_{n}: n<\omega\right\}$ be a countable basis for the topology of $Y$ inherited from $\mathbb{R}$. Let $X=\{0,1\} \times Y$ and for each $n$ let $h_{n}$ be the permutation of $X$ defined as follows:

$$
h_{n}(i, x)= \begin{cases}\langle i, x\rangle & \text { if } x \in U_{n}, \\ \langle 1-i, x\rangle & \text { if } x \in Y \backslash U_{n} .\end{cases}
$$

Let $H$ be the group of permutations of $X$ generated by $\left\{h_{n}: n<\omega\right\}$. Finally, let $\mathcal{A}$ be the $\sigma$-algebra consisting of countable and co-countable subsets of $X$ and $m: \mathcal{A} \rightarrow\{0,1\}$ be the canonical two-valued probability measure defined as follows:

$$
m(A)= \begin{cases}0 & \text { if }|A| \leq \omega \\ 1 & \text { if }|X \backslash A| \leq \omega .\end{cases}
$$

Clearly, $m$ is $H$-invariant and $|H x|=2$ for each $x \in X$.

Let $m^{\prime}: \mathcal{A}^{\prime} \rightarrow[0,1]$ be an $H$-invariant extension of $m$ and suppose that $S \in \mathcal{A}^{\prime}$ for a certain selector $S$ of $H$-orbits. Note that

$$
\left(\{0,1\} \times U_{n}\right) \cap S=h_{n}[S] \cap S \in \mathcal{A}^{\prime} \quad \text { for each } n<\omega .
$$

It follows that if we define

$$
\mu(A)=m^{\prime}((\{0,1\} \times A) \cap S) \quad \text { whenever } \quad(\{0,1\} \times A) \cap S \in \mathcal{A}^{\prime},
$$

then $\mu \mid \mathbf{B}(Y)$ is a finite $\left(\mu(Y)=m^{\prime}(S)=1 / 2\right)$ nonzero Borel measure on $Y$ which vanishes on singletons. This contradicts the choice of $Y$. 
The nonmeasurability of selectors of $H$-orbits in the example above stems from the fact that the measure under consideration cannot be extended to a certain countable collection of subsets of $X$. Our subsequent results show that for measures without this property condition $(\mathbf{V})$ becomes relevant again.

We say that a set $A \in \mathcal{A}$ with $m(A)>0$ is an $H$-atom if it is minimal among the sets $B \in \mathcal{A}$ with $B^{*} \stackrel{m}{=} A^{*}$ in the sense that for any $B \in \mathcal{A}$ the conditions $B^{*} \stackrel{m}{=} A^{*}$ and $B \subseteq A$ imply $m(A \backslash B)=0$ (see [5] and [6]). Following [5] we say that the dynamical system $\langle X, \mathcal{A}, H, m\rangle$ is discrete if there exists an $H$-atom which is an almost complete section for $H$. We need the following well-known fact (see e.g. [5, Theorem III.2 and Proposition VII.1]).

Proposition 4.2. Assume that no $B \in \mathcal{A}$ with $0<m(B)<\infty$ has the property that for every $\varepsilon>0$ there exists a complete section $A \in \mathcal{A}$ for $E_{H} \mid B$ with $m(A)<\varepsilon$. Then the dynamical system $\langle X, \mathcal{A}, H, m\rangle$ is discrete. Moreover, if $A \in \mathcal{A}$ is an $H$-atom with $A^{*} \stackrel{m}{=} X$, then there is a partition of $X$ into $H$-invariant parts $X_{n} \in \mathcal{A}, n \leq \omega$, such that if $m\left(X_{n}\right)>0$ then $X_{n} \stackrel{m}{=} \bigcup_{i<n} A_{n, i}$ where $A_{n, 0}=A \cap X_{n}$ and $\left\{A_{n, i}: i<n\right\}$ is a family of $n$ pairwise disjoint $H$-atoms with $A_{n, i} \sim_{\infty} A_{n, 0}$ for each $i<n$.

Now we are ready to prove the main technical result of this paper.

TheOREM 4.3. Let $H$ be a countable group of permutations of a set $X$ and let $m: \mathcal{A} \rightarrow[0, \infty]$ be a $\sigma$-finite $H$-invariant measure on $X$ such that $m^{*}(\{x \in X:|H x \cap B|=\omega\})=0$ for every $B \in \mathcal{A}$ with $0<m(B)<\infty$. Let $S$ be a selector of $H$-orbits. If there exists an extension of $m$ for which all sets $h[S], h \in H$, are measurable, then there exists an $H$-invariant extension of $m$ for which $S$ is measurable.

Proof. Let $\mathcal{A}^{\prime}$ be the $\sigma$-algebra of subsets of $X$ generated by $\mathcal{A} \cup\{h[S]$ : $h \in H\}$. Since the set of generators of $\mathcal{A}^{\prime}$ is $H$-invariant, so is $\mathcal{A}^{\prime}$. Let $\mu: \mathcal{A}^{\prime} \rightarrow[0, \infty]$ be an arbitrary extension of $m$. With the help of $\mu$ we are going to find an $H$-invariant extension $m^{\prime}: \mathcal{A}^{\prime} \rightarrow[0, \infty]$ of $m$.

By a relativization of Theorem 2.2 and Proposition 4.2, the dynamical system $\langle X, \mathcal{A}, H, m\rangle$ is discrete. Let $A \in \mathcal{A},\left\{X_{n}: n \leq \omega\right\}$, and $\left\{A_{n, i}: i<n\right\}$ have the properties stated in Proposition 4.2.

It suffices to find for every $n \leq \omega$ an extension of $m \mid\left(\mathcal{A} \cap \mathcal{P}\left(A_{n, 0}\right)\right)$ to a measure $m_{n, 0}^{\prime}$ defined on $\mathcal{A}^{\prime} \cap \mathcal{P}\left(A_{n, 0}\right)$ and $H$-invariant in the following sense: whenever $B_{1}, B_{2} \in \mathcal{A}^{\prime} \cap \mathcal{P}\left(A_{n, 0}\right)$ and $B_{1} \sim_{\infty} B_{2}$ in $\mathcal{A}^{\prime}$, then $m_{n, 0}^{\prime}\left(B_{1}\right)=$ $m_{n, 0}^{\prime}\left(B_{2}\right)$. Indeed, suppose that we already have extensions $m_{n, 0}^{\prime}$. For each pair $n \leq \omega$ and $i<n$ let $g_{n, i}$ be a fixed bijection between $A_{n, 0}$ and $A_{n, i}$ which witnesses that $A_{n, 0} \sim_{\infty} A_{n, i}$ in $\mathcal{A}$ (more precisely, $g_{n, i}\left|C_{k}=h_{k}\right| C_{k}$ for $k<\omega$, where $\left\{C_{k}: k<\omega\right\}$ and $\left\{D_{k}: k<\omega\right\}$ are some fixed partitions of 
$A_{n, 0}$ and $A_{n, i}$, respectively, into pieces from $\mathcal{A}$, with $D_{k}=h_{k}\left[C_{k}\right]$ for each $k<\omega$ and some fixed $\left.h_{k} \in H\right)$. Then the measure $m^{\prime}$ defined by

$$
m^{\prime}(A)=\sum_{n \leq \omega} \sum_{i<n} m_{n, 0}^{\prime}\left(g_{n, i}{ }^{-1}\left[A \cap A_{n, i}\right]\right) \quad \text { for } A \in \mathcal{A}^{\prime}
$$

is easily seen to be an $H$-invariant extension of $m$.

Thus, with no loss of generality we assume that $A=A_{n, 0}$ for a fixed $n \leq \omega$. For each $k \leq \omega$ let $A_{k}=\{x \in A:|H x \cap A|=k\}$; clearly, $A=$ $\bigcup_{k \leq \omega} A_{k}$ and the sets $A_{k}$ form a partition of $A$. We claim that $A_{k} \in \mathcal{A}^{\prime}$ or even more: $\{x \in A:|H x \cap B|=k\} \in \mathcal{A}^{\prime}$ for every $B \subseteq A, B \in \mathcal{A}^{\prime}$ and $k \leq \omega$. For if, moreover, $B \neq \emptyset$, then $S \cap B^{*}$ is countably equidecomposable in $\mathcal{A}^{\prime}$ with a subset $T$ of $B$ (see [17, Lemma 1.1]). Hence $T \in \mathcal{A}^{\prime}$ is a partial selector for $E_{H} \mid B$ such that $T \subseteq B$ and it follows that for every $k<\omega$ we have

$$
\{x \in A:|H x \cap B| \geq k+1\}=\{x \in A:|H x \cap(B \backslash T)| \geq k\} .
$$

The statement that $\{x \in A:|H x \cap B| \geq k\} \in \mathcal{A}^{\prime}$ for every $B \in \mathcal{A}^{\prime}$ and $k<\omega$ can now be proved by induction, and then the rest easily follows.

Define the measure $m_{n, 0}^{\prime}$ by putting, for $B \subseteq A, B \in \mathcal{A}^{\prime}$,

$$
m_{n, 0}^{\prime}(B)=\sum_{k=1}^{\infty} \sum_{j=1}^{k} \frac{j}{k} \mu\left(\left\{x \in A:\left|H x \cap\left(B \cap A_{k}\right)\right|=j\right\}\right) .
$$

Note that $m_{n, 0}^{\prime}$ is $H$-invariant in the sense described above. Indeed, assume that $B_{1}, B_{2} \in \mathcal{A}^{\prime} \cap \mathcal{P}(A)$ and $B_{1} \sim_{\infty} B_{2}$ in $\mathcal{A}^{\prime}$. Then $B_{2}=g\left[B_{1}\right]$ for a certain bijection $g: B_{1} \rightarrow B_{2}$ with $g(x) \in H x$ for each $x \in B_{1}$. Hence if $\left|H x \cap\left(B_{1} \cap A_{k}\right)\right|=j$, then $\left|H x \cap\left(B_{2} \cap A_{k}\right)\right|=\left|H x \cap\left(g\left[B_{1}\right] \cap A_{k}\right)\right|=$ $\left|g\left[H x \cap B_{1} \cap A_{k}\right]\right|=j$ for every $k<\omega$ and $j \leq k$.

To complete the proof, it is enough to check that $m_{n, 0}^{\prime}$ extends $m \mid(\mathcal{A} \cap \mathcal{P}(A))$. So let $B \subseteq A, B \in \mathcal{A}$. Since $m$ is $\sigma$-finite it is enough to prove that $m_{n, 0}^{\prime}(B)=m(B)$ under the assumption that $m(B)<\infty$. But then $m^{*}(\{x \in B:|H x \cap B|=\omega\})=0$. At the same time $B \stackrel{m}{=} B^{*} \cap A$, since $A$ is an $H$-atom. Consequently, there are disjoint $H$-invariant sets $C_{1}, C_{2} \in \mathcal{A}$ such that $B \subseteq C_{1} \cup C_{2}, m\left(C_{1}\right)=0, B \cap C_{2}=A \cap C_{2}$ and $\left|H x \cap\left(A \cap C_{2}\right)\right|<\omega$ for every $x \in A \cap C_{2}$. It follows that it suffices to prove that if $C \in \mathcal{A}$ is $H$-invariant, then:

(1) if $m(C)=0$, then $m_{n, 0}^{\prime}(A \cap C)=0$,

(2) if $|H x \cap(A \cap C)|<\omega$ for every $x \in A \cap C$, then $m_{n, 0}^{\prime}(A \cap C)=$ $m(A \cap C)$.

To prove (1), note that $\mu(A \cap C)=m(A \cap C)=0$. 
To prove (2), note that since $C$ is $H$-invariant, $\left|H x \cap\left(C \cap A_{k}\right)\right|=k$ whenever $H x \cap\left(C \cap A_{k}\right) \neq \emptyset$. It follows that

$$
\begin{aligned}
m_{n, 0}^{\prime}(A \cap C) & =\sum_{k=1}^{\infty} \mu\left(A_{k} \cap C\right)=\mu\left(\bigcup_{k=1}^{\infty} A_{k} \cap C\right) \\
& =\mu(A \cap C)=m(A \cap C) .
\end{aligned}
$$

The following two results are immediate corollaries of Theorem 4.3.

THEOREM 4.4. Let $\mathcal{A}$ be a $\sigma$-algebra of subsets of a set $X$ and let $H$ be a countable group of measurable transformations of $X$ such that $\{x \in X:|H x \cap B|=\omega\} \in \mathcal{A}$ for every $B \in \mathcal{A}$ with $0<m(B)<\infty$. Let $m: \mathcal{A} \rightarrow[0, \infty]$ be a $\sigma$-finite $H$-invariant measure on $X$ which can be extended to any countable collection of subsets of $X$. Then the following are equivalent:

(1) there is $B \in \mathcal{A}$ such that $0<m(B)<\infty$ and $|H x \cap B|=\omega$ for every $x \in B$,

(2) every selector of $H$-orbits is nonmeasurable with respect to any $H$ invariant extension of $m$,

(3) there exists a selector of $H$-orbits nonmeasurable with respect to any $H$-invariant extension of $m$.

TheOREM 4.5. Let $H$ be a countable group of permutations of a set $X$ such that all $H$-orbits are finite. Let $m: \mathcal{A} \rightarrow[0, \infty]$ be a $\sigma$-finite $H$-invariant measure on $X$ which can be extended to any countable collection of subsets of $X$. Then for every selector of $H$-orbits there exists an $H$-invariant extension of $m$ for which $S$ is measurable.

Recall that the action of $H$ is $m$-free if $m^{*}(\{x \in X: h(x)=x\})=0$ for any $h \in H \backslash\left\{\operatorname{id}_{X}\right\}$. Under this assumption we get the equivalences from Theorem 4.4 without any additional hypotheses.

TheOREM 4.6. Let $m: \mathcal{A} \rightarrow[0, \infty]$ be a $\sigma$-finite measure on $X$ invariant under a countable group $H$ of measurable transformations of $X$ such that the action of $H$ on $X$ is $m$-free. Then the following are equivalent:

(1) there is $B \in \mathcal{A}$ such that $0<m(B)<\infty$ and $|H x \cap B|=\omega$ for every $x \in B$,

(2) every selector of $H$-orbits is nonmeasurable with respect to any $H$ invariant extension of $m$,

(3) there exists a selector of $H$-orbits nonmeasurable with respect to any $H$-invariant extension of $m$.

Proof. To see that (1) implies (2), argue as in the proof of Proposition 1.1. Then the only nontrivial implication left is $(3) \Rightarrow(1)$ or, equivalently, $\neg(1) \Rightarrow \neg(3)$. So assume that there is no $B \in \mathcal{A}$ with $0<m(B)<\infty$ and such 
that $|H x \cap B|=\omega$ for every $x \in B$. Let $S$ be a selector of $H$-orbits. With no loss of generality assume that the measure $m$ is complete. In particular, $\{x \in X: h(x)=x\} \in \mathcal{A}$ for each $h \in H$.

In order to prove the existence of an $H$-invariant extension of $m$ for which $S$ is measurable, it suffices to check the hypotheses of Theorem 4.3.

To prove that $m(\{x \in X:|H x \cap B|=\omega\})=0$ for every $B \in \mathcal{A}$ with $0<m(B)<\infty$, note that for each $B \in \mathcal{A}$ and $x \in X$,

$$
\begin{aligned}
& |H x \cap B|<\omega \\
& \quad \Leftrightarrow \exists F \subseteq H(|F|<\omega \wedge \forall h \in H(h(x) \in B \Rightarrow \exists f \in F h(x)=f(x))) .
\end{aligned}
$$

This implies that $\{x \in X:|H x \cap B|<\omega\} \in \mathcal{A}$. Since, moreover, for every $B \in \mathcal{A}$ with $0<m(B)<\infty$ there is $x \in B$ with $|H x \cap B|<\omega$, the claim follows.

Finally, to prove that there exists an extension of $m$ for which all sets $h[S], h \in H$, are measurable, note that there is an $H$-invariant set $Z \in \mathcal{A}$ such that $m(Z)=0$ and the sets $h[Z] \backslash Z$ form a partition of $X \backslash Z$. But, by a theorem of Bierlein (see [4] or [1]), one can always extend a measure so that all members of a given countable partition of a measurable set are in the domain of the extension.

Finally, the following result shows that in general we cannot strengthen Theorem 4.4 by removing the additional measurability condition from its hypotheses.

EXAMPle 4.7. Assume that there is no universally measure zero set of cardinality continuum (by a theorem of Miller [9], this assumption is consistent with the usual axioms of set theory). There exist a set $X$ and a probability measure $m: \mathcal{A} \rightarrow[0,1]$ invariant with respect to a countable group $H$ of permutations of $X$ and having the following properties:

(1) $m$ can be extended to any countable collection of subsets of $X$,

(2) $m^{*}(\{x \in X:|H x|<\omega\})=1$,

(3) every selector of $H$-orbits is nonmeasurable with respect to any $H$ invariant extension of $m$.

Proof. Let $X_{1}=\{0,1\} \times Y$ where $Y \subseteq \mathbb{R}$ is an uncountable universally measure zero set. Let $H_{1}$ be the group of permutations of $Y_{1}$ constructed in Example 4.1.

Let $X_{2}$ be an arbitrary set of cardinality continuum disjoint from $X_{1}$. Let $H_{2}$ be a countable group of permutations of $X_{2}$ with infinite $H_{2}$-orbits.

Let $X=X_{1} \cup X_{2}, \mathcal{A}$ be the $\sigma$-algebra consisting of countable and cocountable subsets of $X$ and $m: \mathcal{A} \rightarrow\{0,1\}$ be the canonical two-valued probability measure on $\mathcal{A}$ (see Example 4.1). 
Finally, let $H$ consist of permutations $h$ of $X$ such that $h \mid X_{1} \in H_{1}$ and $h \mid X_{2} \in H_{2}$.

Clearly, $m$ is an $H$-invariant probability measure on $X$.

To prove property (1), let $\left\{A_{n}: n<\omega\right\}$ be a collection of subsets of $X$. Since there is no universally measure zero set of cardinality continuum, there is a probability measure $m_{2}$ defined on a $\sigma$-algebra $\mathcal{A}_{2}$ of subsets of $X_{2}$ containing all sets of the form $A_{n} \cap X_{2}$ and such that all singletons have measure $m_{2}$ zero. Then the measure $\mu$ on $X$ defined by

$$
\mu(A)=m_{2}\left(A \cap X_{2}\right) \quad \text { whenever } \quad A \cap X_{2} \in \mathcal{A}_{2}
$$

extends $m$ and measures all the $A_{n}$ 's.

Property (2) is obvious, since $\{x \in X:|H x|<\omega\}=X_{1}$.

To prove property $(3)$, let $m^{\prime}: \mathcal{A}^{\prime} \rightarrow[0,1]$ be an $H$-invariant extension of $m$. Since any $H$-invariant measure can always be extended in an invariant way by a single $H$-invariant set (this is essentially due to Szpirlajn [15]; see also [19, Section 3]), with no loss of generality assume that $X_{1}, X_{2} \in \mathcal{A}^{\prime}$. Then there is $i \in\{0,1\}$ such that $m^{\prime}\left(X_{i}\right)>0$ and $S \cap X_{i}$ is a measurable selector of $H_{i}$-orbits. To reach a contradiction argue either as in Example 4.1 (if $i=1$ ) or as in Proposition 1.1 (if $i=2$ ).

\section{References}

[1] A. Ascherl and J. Lehn, Two principles for extending probability measures, Manuscripta Math. 21 (1977),43-50.

[2] S. Banach et A. Tarski, Sur la décomposition des ensembles de points en parties respectivement congruents, Fund. Math. 6 (1924), 244-277.

[3] H. Becker and A. S. Kechris, The Descriptive Set Theory of Polish Group Actions, London Math. Soc. Lecture Note Ser. 232, Cambridge Univ. Press, 1996.

[4] D. Bierlein, Über die Fortsetzung von Wahrscheinlichkeitsfeldern, Z. Wahrsch. Verw. Gebiete 1 (1962), 28-46.

[5] Dang-Ngoc-Nghiem, On the classification of dynamical systems, Ann. Inst. H. Poincaré Sect. B 9 (1973), 397-425.

[6] Y. Kawada, Über die Existenz der invarianten Integrale, Japan. J. Math. 19 (1944), 81-95.

[7] A. S. Kechris, Classical Descriptive Set Theory, Grad. Texts in Math. 156, Springer, 1995.

[8] A. B. Kharazishvili and A. P. Kirtadze, On weakly metrically transitive measures and nonmeasurable sets, Real Anal. Exchange 32 (2007), 553-562.

[9] A. W. Miller, Mapping a set of reals onto the reals, J. Symbolic Logic 48 (1983), $575-584$.

[10] - Special subsets of the real line, in: Handbook of Set-Theoretic Topology, NorthHolland, 1984, 201-233.

[11] H. Minkowski, Geometrie der Zahlen, Teubner, Leipzig, 1896.

[12] M. G. Nadkarni, On the existence of a finite invariant measure, Proc. Indian Acad. Sci. Math. Sci. 100 (1990), 203-220. 
[13] S. Solecki, On sets nonmeasurable with respect to invariant measures, Proc. Amer. Math. Soc. 119 (1993), 115-124.

[14] —, Measurability properties of sets of Vitali's type, ibid., 897-902.

[15] E. Szpilrajn, Sur l'extension de la mesure lebesguienne, Fund. Math. 25 (1935), 551-558.

[16] G. Vitali, Sul problema della misura dei gruppi di punti di una retta, Gamberini e Parmeggiani, Bologna, 1905.

[17] P. Zakrzewski, When do equidecomposable sets have equal measures?, Proc. Amer. Math. Soc. 113 (1991), 831-837.

[18] - , The existence of invariant $\sigma$-finite measures for a group of transformations, Israel J. Math. 83 (1993), 275-287.

[19] —, Measures on algebraic-topological structures, in: Handbook of Measure Theory, E. Pap (ed.), North-Holland, 2002, 1091-1130.

Institute of Mathematics

University of Warsaw

Banacha 2

02-097 Warszawa, Poland

E-mail: piotrzak@mimuw.edu.pl 